\title{
Controllable formation of periodic wrinkles in Marangoni-driven self-assembled graphene film for sensitive strain detection
}

\author{
Yufei Jia ${ }^{1 \dagger}$, Wenjun Chen ${ }^{1 \dagger}$, Chen $\mathrm{Ye}^{2}$, Rongliang Yang ${ }^{1}$, Leilei Yang ${ }^{1}$, Zian Zhang ${ }^{1}$, Qingmei Hu ${ }^{1}$, \\ Binghao Liang ${ }^{1}$, Bo-Ru Yang ${ }^{1}$, Zikang Tang ${ }^{1,3}$, Cheng-Te Lin $^{2^{*}}$ and Xuchun Gui ${ }^{1 *}$
}

\begin{abstract}
Controllable formation of microstructures in the assembled graphene film could tune the physical properties and broaden its applications in flexible electronics. Many efforts have been made to control the formation of wrinkles and ripples in graphene films. However, the formation of orderly wrinkles in graphene film remains a challenge. Here, we reported a simple strategy for the fabrication of graphene film with periodic and parallel wrinkles with a pre-stretched polydimethylsiloxane substrate. The width of the wrinkles in graphene can be controlled by changing the pre-stretched strain of the substrate. The average width of wrinkles in graphene film on the substrate with pre-stretched strain of $10 \%$, $20 \%$, and $50 \%$ was about $3.68,2.99$ and $2.01 \mu \mathrm{m}$, respectively. The morphological evolution of wrinkled double-layered graphene under mechanical deformation was observed and studied. Furthermore, a strain sensor was constructed based on the wrinkled graphene, showing high sensitivity, large working range and excellent cyclic stability. These strain sensors show great potential in real-time motion detection, health surveillance and electronic skins.
\end{abstract}

Keywords: wrinkled double-layered structure, solution-processed, assembled graphene film, Marangoni effect, strain sensor

\section{INTRODUCTION}

Graphene is an ideal material to be applied in wearable and flexible sensors and detectors due to its outstanding electrical and mechanical properties [1-4]. Particularly, mechanical sensors based on large-area graphene films have great application potential for integration in electronic devices, which could promote the development of the internet of things [5-9]. Chemical vapor deposition is a common method to synthesize large-area and highquality graphene films, but the high cost and complicated transfer procedures limit their scalable fabrication and massive applications [10-12]. Besides, liquid-phase exfoliation is a more effective and low-cost method to produce graphene derivative solutions [13]. These solutions can be used to assemble graphene films by the methods of spraying [14], spin coating [15], drop casting [16] and laser scribing [17] for the applications in wearable mechanical sensors. However, for these methods, special conditions such as expensive equipment and complicated process are usually required. On the contrary, a facile interfacial assembly method for the fabrication graphene films has been proposed in recent years. According to this approach, graphene nanosheets/ethanol solution was injected into deionized (DI) water to selfassemble large-size graphene film according to the Marangoni effect $[18,19]$.

In addition, the artificial formation of wrinkles or ripples in graphene films could lead to novel applications such as high-performance wearable and flexible electronics [20,21]. Several methods based on different mechanisms have been used to fabricate graphene films with wrinkled structures. Recently, we proposed a solutionprocessed shrinking method to accomplish the controllable fabrication of homogeneous wrinkles in large-

\footnotetext{
${ }^{1}$ State Key Laboratory of Optoelectronic Materials and Technologies, School of Electronics and Information Technology, Sun Yat-sen University, Guangzhou 510275, China

${ }^{2}$ Key Laboratory of Marine Materials and Related Technologies, Zhejiang Key Laboratory of Marine Materials and Protective Technologies, Ningbo Institute of Materials Technology and Engineering (NIMTE), Chinese Academy of Sciences, Ningbo 315201, China

${ }^{3}$ Institute of Applied Physics and Materials Engineering, University of Macau, Avenida da Universidade, Taipa, Macau 999078, China

${ }^{\dagger}$ These authors contributed equally to this work.

* Corresponding authors (emails: guixch@mail.sysu.edu.cn (Gui X); linzhengde@nimte.ac.cn (Lin CT))
} 
area graphene films [22]. However, the created wrinkles were randomly distributed in the graphene and difficult to be periodic. In contrast, there is a common method to develop periodically organized wrinkles or defined wrinkled patterns by transferring graphene onto flexible polymer substrates, which mainly rely on the mechanical deformation of the polymers. It is exemplified that graphene and its derivatives could be deposited onto prestretched polymer substrates to prepare well-designed wrinkles by controlling the pre-stretched strain and releasing direction of the substrate [23-25]. Because some kinds of thermoplastics such as polystyrene may shrink at high temperature, graphene films can also form patterned structures by transferring it on these polymers, followed by thermal treatment [26-29]. In these methods, efforts have been made to further improve the physical properties of graphene films and apply them in flexible electronic devices. However, the precise control of wrinkled morphology in graphene films and morphological evolution of wrinkles under mechanical deformation has not been adequately exploited.

Herein, we reported a solution-processed method for the fabrication of large-area graphene films with precisely controlled periodic/parallel wrinkles and double-layered structure. The wrinkled graphene film was fabricated by an interfacial self-assembly process, followed by being transferred onto an uniaxial pre-stretched polydimethylsiloxane (PDMS) substrate. The width of the wrinkles can be adjusted by controlling the pre-stretched strain of the substrate. What's more, the deformation mechanism of wrinkled double-layered graphene under tensile strain was investigated, and the results indicated that, during stretching, the bottom layer wrinkles were gradually flattened even cracked, while the top wrinkled graphene kept continuous. A highly-sensitive strain sensor based on the wrinkled double-layered graphene was fabricated, showing good prospects to be applied in flexible electronics for motion detection, health surveillance and artificial skins.

\section{EXPERIMENTAL SECTION}

\section{Strain sensor fabrication}

First, graphene nanosheets were prepared by the electrochemical exfoliation method, as reported by our previous study [18]. Graphene nanosheets were peeled from graphite electrode and then formed a graphene/ethanol solution. Then, $5 \mathrm{~mL}$ of graphene nanosheets/ethanol solution with a concentration of $0.5 \mathrm{mg} \mathrm{mL}^{-1}$ was dripped dropwise onto the surface of deionized (DI) water at a rate of $0.1 \mathrm{~mL} \mathrm{~s}^{-1}$ to prepare the assembled graphene film. A PDMS substrate with the thickness of $200 \mu \mathrm{m}$ was treated in plasma for $4 \mathrm{~min}$ in advance to improve its hydrophilicity. Subsequently, the graphene film was "fished out" by the pre-stretched PDMS substrate, which was stretched and fixed on a small and customized manual displacement stage (Fig. S1), and dried at $50^{\circ} \mathrm{C}$ for $30 \mathrm{~min}$. In order to obtain wrinkled double-layered graphene film, the transfer procedure was repeated once more and the formerly transferred graphene film was overlapped by the latter one. By gradually releasing the pre-stretched PDMS substrate, wrinkled double-layered graphene film was obtained. Afterwards, the graphene film was annealed in the mixture of $\mathrm{Ar} / \mathrm{H}_{2}$ for $5 \mathrm{~h}$ at $200^{\circ} \mathrm{C}$. Finally, two copper wires were connected at both ends of the graphene film using silver paint to fabricate strain sensors with $2 \times 0.5 \mathrm{~cm}^{2}$ in area.

\section{Characterizations}

Scanning electron microscopy (SEM) images were captured by using a Hitachi S-4800 SEM. For capture of SEM images of wrinkled graphene films under tension, the sample was fixed on the displacement stage (Fig. S1), which was directly installed on the sample stage of SEM. Optical microscopy images were taken by a Zeiss Axio CSM 700. Raman spectra were collected by a HORIBA JY HR 800 and the wavelength of the incident laser is $633 \mathrm{~nm}$. Atomic force microscopy (AFM) characterizations were performed using a Veeco Edge. An electric displacement stage (Zolix TSA100) with the resolution of $1.25 \mu \mathrm{m}$ was employed to control the loading and unloading strain on the sensors, during which the electromechanical responses were recorded by a Keithley 2400 digital multimeter.

\section{RESULTS AND DISCUSSION}

The preparation process of the wrinkled graphene film is illustrated in Fig. 1a. First, graphene nanosheets/ethanol solution was dripped onto the surface of DI water. Due to the Marangoni effect, by which the interfacial surface tension between the drop and DI water suddenly decreased because of the lower surface tension of ethanol $\left(22.3 \mathrm{mN} \mathrm{m}^{-1}\right)$ than DI water $\left(72.7 \mathrm{mN} \mathrm{m}^{-1}\right)$ [22], graphene/ethanol solution would rapidly diffuse on the surface of DI water, which motivates graphene nanosheets to move, collide and overlap with each other, eventually form a continuous graphene film [18]. Then a target substrate, such as PDMS was used to "fish out" the assembled graphene film. In contrast to the methods of spraying [30] and laser scribing [31], this interfacial as- 

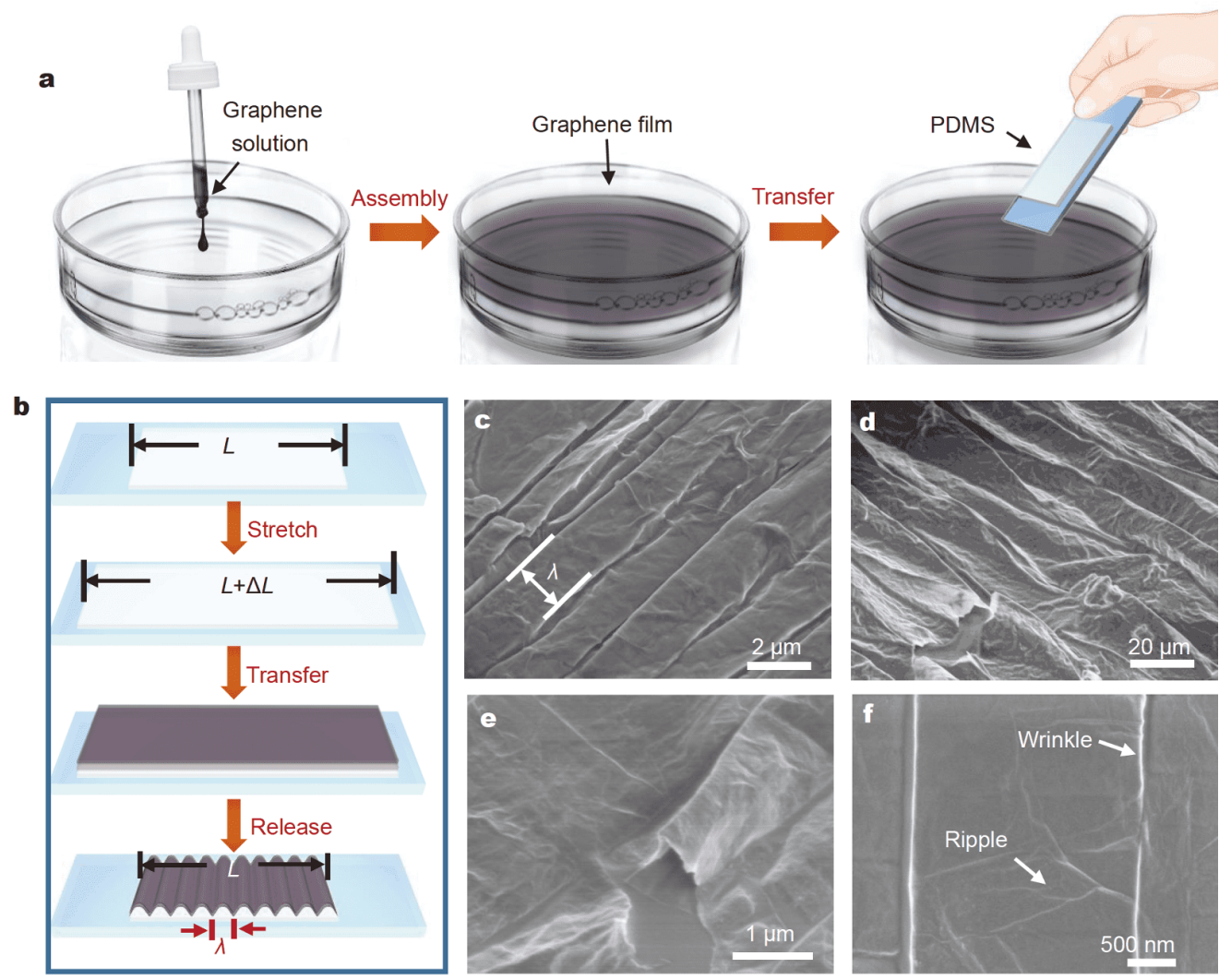

Figure 1 Controllable fabrication of wrinkled graphene. (a) Schematic illustration of the fabrication of graphene films by interface assembly. (b) Schematic illustration of the pre-stretched PDMS substrate for the fabrication of wrinkled graphene films. (c, d) SEM images of the wrinkled graphene, showing periodic and orderly wrinkled structure in the film. (e) High magnification SEM image of a wrinkle, showing the double-layer structure. (f) SEM image of wrinkled graphene with ripples and wrinkles.

sembly method is simple, efficient, time-saving, and easeof-scale up. More importantly, in comparison to similar materials produced by the traditional methods, such as drop-casting and spin-coating processes, the self-assembled films driven by the Marangoni force show much higher structural homogeneity $[18,32]$. In order to fabricate graphene film with controlled wrinkled structures, a PDMS substrate with the length of $L$ was uniaxially stretched to $L+\Delta L$, as shown in Fig. 1b. After "fishing out" the assembled graphene film on the pre-stretched PDMS substrate and the release of the PDMS to the initial length $(L)$, the graphene film was subjected to stress in the releasing direction to form unidirectional periodic wrinkles. The pre-stretched PDMS with the strain $(\Delta L / L)$ of $0 \%$, $10 \%, 20 \%$, and $50 \%$ were used as the substrate. These wrinkled graphene samples were denoted as pre-strain-0, pre-strain-10\%, pre-strain-20\%, and pre-strain-50\%, respectively. Wavelength $(\lambda)$ marked in Fig. $1 \mathrm{~b}$ was used to define the width between the two wrinkles.

SEM image (Fig. 1c) confirms the formation of periodic graphene wrinkles with a width of $\lambda$. Due to the Poisson effect, the expansion in the orthogonal direction could induce some small cracks in the wrinkled graphene film (Fig. 1d). The transfer step can be repeated to continuously stack wrinkled graphene films. For instance, the SEM image in Fig. 1e clearly illustrates the formation of double layers of wrinkled structures in the graphene film after twice of transfer. Additionally, the thickness of the wrinkled graphene film is doubled or tripled after twice or thrice of transfer, respectively (Fig. S2). Therefore, it is feasible to control the thickness of the wrinkled graphene film with this method. It is worth noticing that during the transfer process, random ripples between two parallel wrinkles were generated inevitably (Fig. 1f). The formation of the random ripples should be caused by the evaporation of the water trapped in the graphene film and the interaction between the graphene film and PDMS substrate [33]. Although the shrink-induced wrinkled graphene oxide film (more than $\sim 1 \mu \mathrm{m}$ in thickness) could maintain its free-standing structure after the sup- 
porting substrate is etched [34], the wrinkled graphene film in this study may be broken into fragments without the support of PDMS substrate, because the thickness of this film is less than $200 \mathrm{~nm}$ (Fig. S2). We did the following experiments with the wrinkled double-layered graphene film prepared on the pre-stretched PDMS substrates.

The morphology of the wrinkled graphene can be precisely tuned by changing the pre-strain $\left(\varepsilon_{\text {pre }}\right)$ of the PDMS substrate, which is defined as $\varepsilon_{\text {pre }}=\Delta L / L$. When the graphene film was assembled on the PDMS substrate without pre-strain $\left(\varepsilon_{\text {pre }}=0 \%\right)$, small random ripples and no wrinkles can be observed in the sample, as exhibited in Fig. 2a, b. The formation of these ripples is attributed to the evaporation of the water encapsulated in the graphene film and the interactions between the graphene film and the elastomer substrate $[19,33,35]$. Hence, these ripples distribute randomly and uncontrollably. On the contrary, the directional and ordered wrinkles form in the graphene film, as the films were fabricated by the prestretched PDMS substrates with $\varepsilon_{\text {pre }}=10 \%-50 \%$, as shown in Fig. 2c-h. Furthermore, with an increase of $\varepsilon_{\text {pre }}$, $\lambda$ of the wrinkles reduces. The wrinkled graphene on the pre-stretched PDMS substrate with $\varepsilon_{\text {pre }}=50 \%$ has the smallest $\lambda$ (Fig. 2g, h). Optical microscope images (Fig. S3) further confirm the uniform distribution of graphene wrinkles over a large area, and Fig. S4 provides the morphological details of the wrinkled graphene film.

For quantitative analysis of wrinkle distributions in the graphene films, 200 random positions from each sample were selected to perform statistical analysis of $\lambda$. Accordingly, the average $\lambda$ of wrinkles for the sample of prestrain-10\%, pre-strain-20\%, and pre-strain-50\% is $3.68 \pm$ $0.59,2.99 \pm 0.65$ and $2.01 \pm 0.57 \mu \mathrm{m}$, respectively (Fig. $2 \mathrm{i}-$
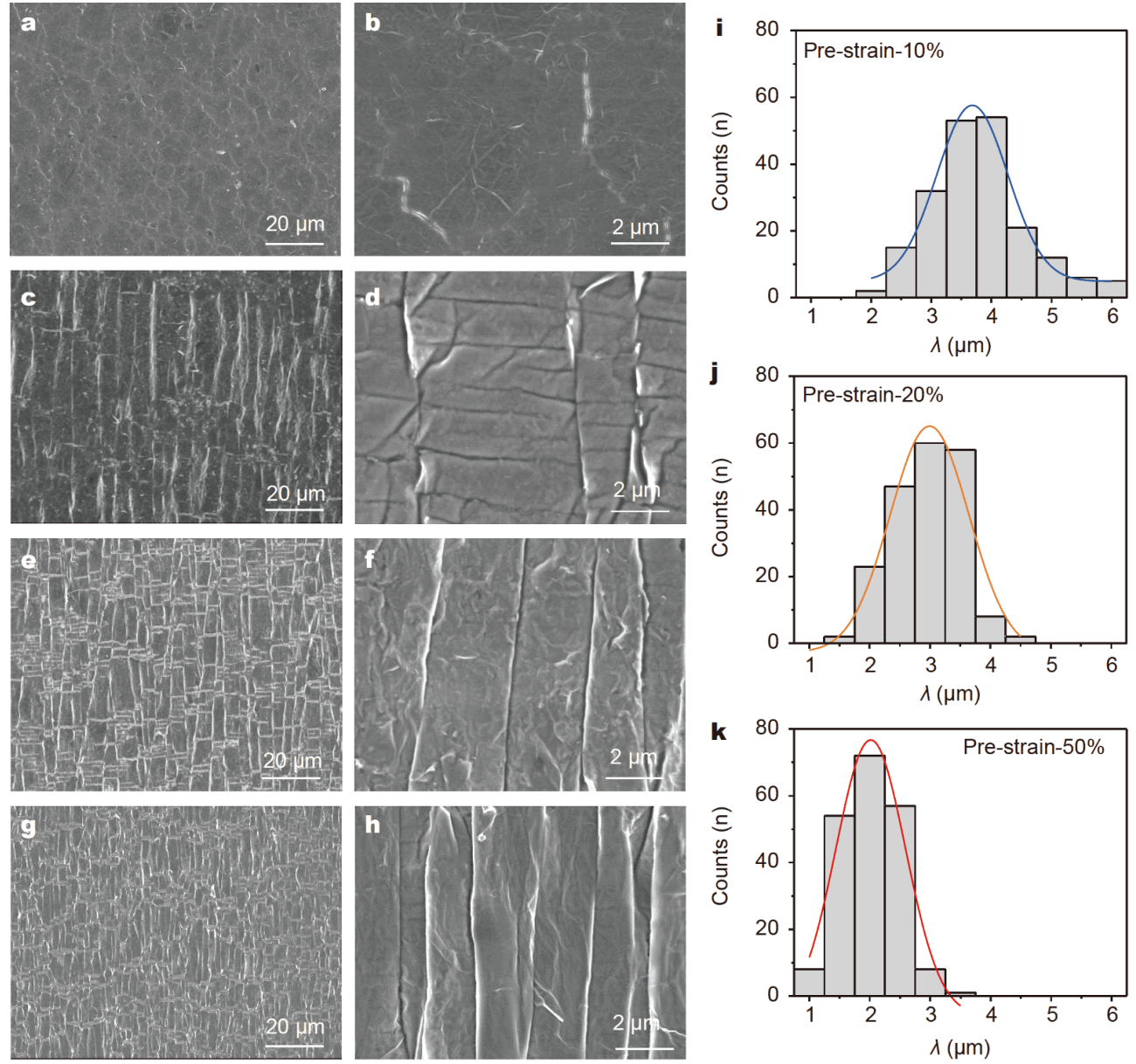

Figure 2 Morphology characterization of the wrinkled graphene films. (a, b) SEM images of the wrinkled graphene film based on the non-stretched PDMS substrate (pre-strain-0). SEM images of wrinkled graphene film based on the pre-stretched PDMS substrates with strain of $10 \%$ (pre-strain$10 \%)(\mathrm{c}, \mathrm{d})$, pre-strain-20\% (e, f), and pre-strain-50\% (g, h). Histogram and corresponding gauss fit (solid curve) of wrinkles' wavelength distribution of the graphene films, (i) pre-strain- $10 \%$, (j) pre-strain- $20 \%$, and (k) pre-strain- $50 \%$, respectively. 
k). It indicates that there is an inverse relationship between $\varepsilon_{\text {pre }}$ and $\lambda$. Theoretically, $\lambda$ can be calculated by [36]:

$\lambda=\frac{h}{\varepsilon_{0}^{1 / 2}}\left(\frac{\mu_{\mathrm{F}}}{\mu_{\mathrm{S}}}\right)^{1 / 3}[\mu \mathrm{m}]$,

where $h$ is the thickness of the graphene film measured by AFM (Fig. S2), $\varepsilon_{0}$ is equal to $(L+\Delta L) / L_{0}$, namely $1+\varepsilon_{\text {pre, }}$, and $\mu_{\mathrm{F}}$ and $\mu_{\mathrm{S}}$ represent the shear modulus of graphene film and PDMS substrate, which are about $4 \mathrm{GPa}$ [37] and $0.46 \mathrm{MPa}$ [36] from the early reports, respectively. According to the above equation, $\lambda$ of wrinkles for the substrates with $\varepsilon_{\text {pre }}=10 \%, 20 \%$, and $50 \%$ were calculated to be $2.4-3.7,2.2-3.4$ and $1.8-2.7 \mu \mathrm{m}$, respectively, in good agreement with the experimental values. Consequently, the experimental and calculation results evidence the high feasibility of our proposed method to fabricate unidirectional wrinkled structures in graphene films.

The precise control of wrinkle formation paves an effective way to tune the physical properties of graphene film. As shown in Fig. 3a, the graphene film (pre-strain-0) with no wrinkles exhibits high optical transmittance of $83.9 \%$ at $550 \mathrm{~nm}$. Although the construction of wrinkled structures slightly decreases its transparency, the trans- mittance of wrinkled graphene film formed on the prestretched substrate with $\varepsilon_{\text {pre }}=50 \%$ still maintains more than $74 \%$, which is comparable to that of its counterparts reported previously $[20,38]$. What's more important, its transmittance can be increased to $81.7 \%$, when the sample is under $50 \%$ tensile strain in the direction across the wrinkles. The formation of wrinkled morphology reduces the transparency of graphene film but improves its electrical conductivity (Fig. 3b). As the pre-stretched strain of the substrate increases, the resistance of the sample decreases. The two-terminal resistance of the wrinkled graphene (pre-strain-50\%) is about $441 \mathrm{k} \Omega$. The reason is that the formation of wrinkles increases the overlapping area between graphene layers and creates more vertical conductive pathways to promote electrical conduction, resulting in the reduction of the resistance [22,39]. Additionally, the linear $I-V$ curves (inset of Fig. $3 \mathrm{~b}$ ) indicate an excellent Ohmic characteristic of the wrinkled graphene.

Fig. $3 \mathrm{c}$ illustrates the structural changes of the wrinkled double-layered graphene under tensile strain for the explanation of the deformation and fracture mechanism. The wrinkled double-layered graphene formed on the pre-stretched substrate with $\varepsilon_{\text {pre }}=50 \%$ was taken as an
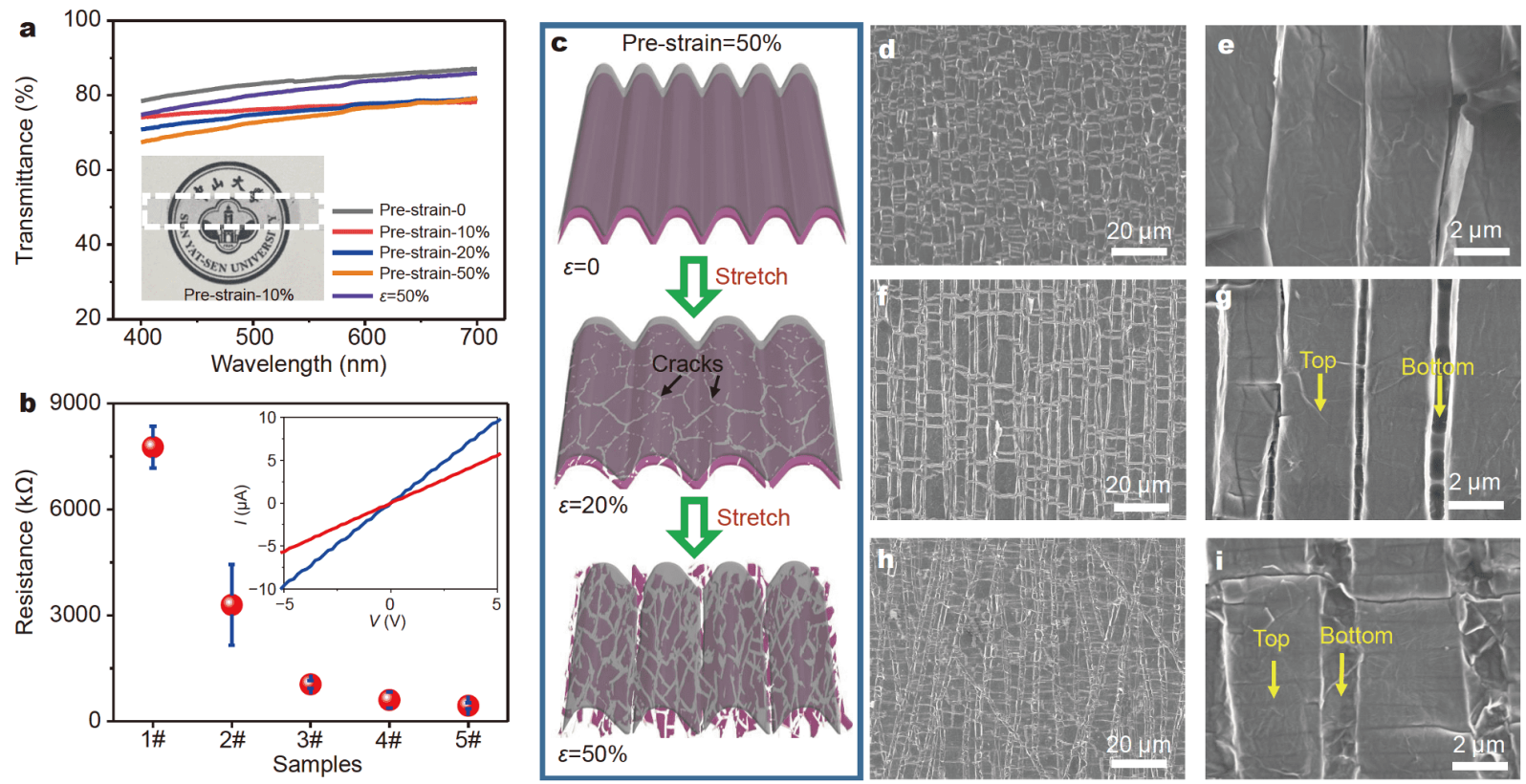

Figure 3 Optical, electrical and mechanical properties of the wrinkled graphene films. (a) Transmittance spectra of different wrinkled graphene samples. Inset is the photograph of wrinkled graphene film of pre-strain-10\%. (b) Resistance of different wrinkled graphene samples. 1\#-5\# samples correspond to single-transferred graphene film on PDMS substrate without pre-stretch, pre-strain- 0 , pre-strain- $10 \%$, pre-strain- $20 \%$, pre-strain- $50 \%$, respectively. Inset is the $I-V$ characteristic curve of the wrinkled graphene films with pre-strain-20\% (red solid line) and pre-strain-50\% (blue solid line), respectively. (c) Schematic illustrating the structure evolution of the wrinkled graphene films under tensile strains of $0 \%, 20 \%$ and $50 \%$. SEM images corresponding to the strain of $0 \%$ in (c) (d, e), $20 \%$ in (c) (f, g), and $50 \%$ in (c) (h, i). 
example for discussion. At the initial state (without tensile strain), the graphene film with one-dimensional uniform wrinkles can be observed (Fig. 3d, e). When it is stretched to $20 \%$, the wrinkles in the bottom layer film are gradually flattened, and $\lambda$ becomes larger until some cracks appear (Fig. 3f, g). In contrast, the wrinkled graphene film at the top layer maintains continuity without obvious damage. Increasing $\varepsilon$ to $50 \%$, the wrinkles at the bottom layer are further cleaved to fragments. Meanwhile, the upper wrinkles are significantly released with a higher $\lambda$ and the cracks are few (Fig. 3h, i). More relevant high-magnification SEM images were captured to indicate the two layers of graphene film under different $\varepsilon$, as shown in Fig. S5. Accordingly, there are different morphological changes of two layers of graphene films under the same tension. Because the formation of wrinkled double-layered graphene is ascribed to the different surface properties in twice of transfer. For the first transfer, the substrate is PDMS, while for the second transfer, the substrate is the former graphene film with random ripples. These ripples prevent the conformal stacking between the two layers of graphene. In comparison with the strong adhesion between the bottom layer of graphene film and PDMS substrate, this weaker interaction between those two layers graphene allows the relative sliding with respect to each other. The simulation results of the different interactivities in two interfaces have been revealed by a previous report [40]. During the stretching process, Raman spectra (Fig. S6) were employed to confirm no apparent shift of G-band of graphene film. It illustrates that the tensile strain primarily induces an interlayer sliding rather than the damages of wrinkled graphene film at the top layer.

Revealing the structural evolution of wrinkled graphene film under mechanical deformation helps design the strain sensors. Detection range and sensitivity are two important indexes to evaluate the performance of sensors for strain detection. In this work, the working range of the sensors based on the wrinkled graphene film can be adjusted by the pre-strain amount of the corresponding PDMS substrate. Gauge factor (GF) is a factor to estimate the sensitivity of mechanical sensors and defined as GF $=$ $\delta R / \varepsilon$ and $\delta R=\Delta R / R_{0}$ [41], where $\varepsilon$ is the strain of the sensor, and $\Delta R$ and $R_{0}$ are the resistance change under strain and original resistance of the sensors, respectively. As plotted in Fig. 4a, the detection range of the sensors expands with increasing pre-strain of PDMS substrates. As expected, the strain sensor based on the wrinkled double-layered graphene formed on the pre-stretched substrate with $\varepsilon_{\text {pre }}=50 \%$ has the widest working range.
However, its GF of 37.1 at an applied strain of $50 \%$ is the lowest among the sensors with different wrinkled morphologies. In contrast, under an applied strain of 5\%, the sensors based on the graphene film without wrinkles (pre-strain-0) possess a nine times higher GF of 348.6, which is superior to that of the counterparts reported previously at the same strain condition [42-44]. Fig. 4b offers details of the performance of the strain sensor based on the sample of pre-strain- 0 at different applied strains, presenting that the $\Delta R / R_{0}$ is relatively small under $2.5 \%$ tensile strain but sharply increases to about $2000 \%$ under the strain of $5.0 \%$. In comparison, although the resistance response of the sensors of pre-strain-50\% under the strain of $5 \%$ is low $(\approx 40 \%)$ in Fig. 4 c, it is capable of operating at a much wider range (with $\Delta R / R_{0}$ of 1300 under $20 \%$ strain), and also has high reliability to distinguish the applied strain (Fig. 4d). It is further clarified in Fig. 4e that the sensor based on the wrinkled graphene film with longer $\lambda$ exhibits higher $\Delta R / R_{0}$ under the same tensile strain. Therefore, the strain sensors based on the wrinkled double-layered graphene film can achieve a good balance between detection range and sensitivity, compared with other similar devices $[14,16,18,21,31,45-$ 48], as shown in Fig. 4f. Moreover, in Fig. 4g, the performance of the strain sensors keeps stable after 5000 stretching/releasing cycles at the strain of $20 \%$, suggesting excellent cyclic stability. After cycling, it has the similar unidirectional wrinkles with a certain wavelength to its initial state (Fig. S7a). The wavelength is $1.76 \pm 0.39 \mu \mathrm{m}$ (Fig. S7), which is slightly lower than its initial value of $2.01 \pm 0.57 \mu \mathrm{m}$ (Fig. 2k).

The adjustable detection range and high repeatability of our sensors based on the wrinkled double-layered graphene films are suitable for practical applications. In order to detect human motions, our strain sensor was installed on the joints of a forefinger, and the bending angles of which were clearly reflected by distinct electrical signals in Fig. 5a. Moreover, the rapid development of mechanical sensors meets the growing demand for health surveillance. In Fig. 5b, by pasting our sensors on a wrist of a volunteer, the resistance responses are in good agreement with the pulse beats with a normal frequency of $\approx 1 \mathrm{~Hz}$. Finally, our strain sensors also show great potential in electronic skins (E-skins). The sensor was fixed on a robot's knee to monitor the dynamic responses during the robot's step-to-retract processes with a maximum deformation of about $45 \%$ (Fig. 5c, d). As a result, our strain sensor is a promising candidate for integrating smart medical instruments to detect vital signs of humans and further offering medical information. 

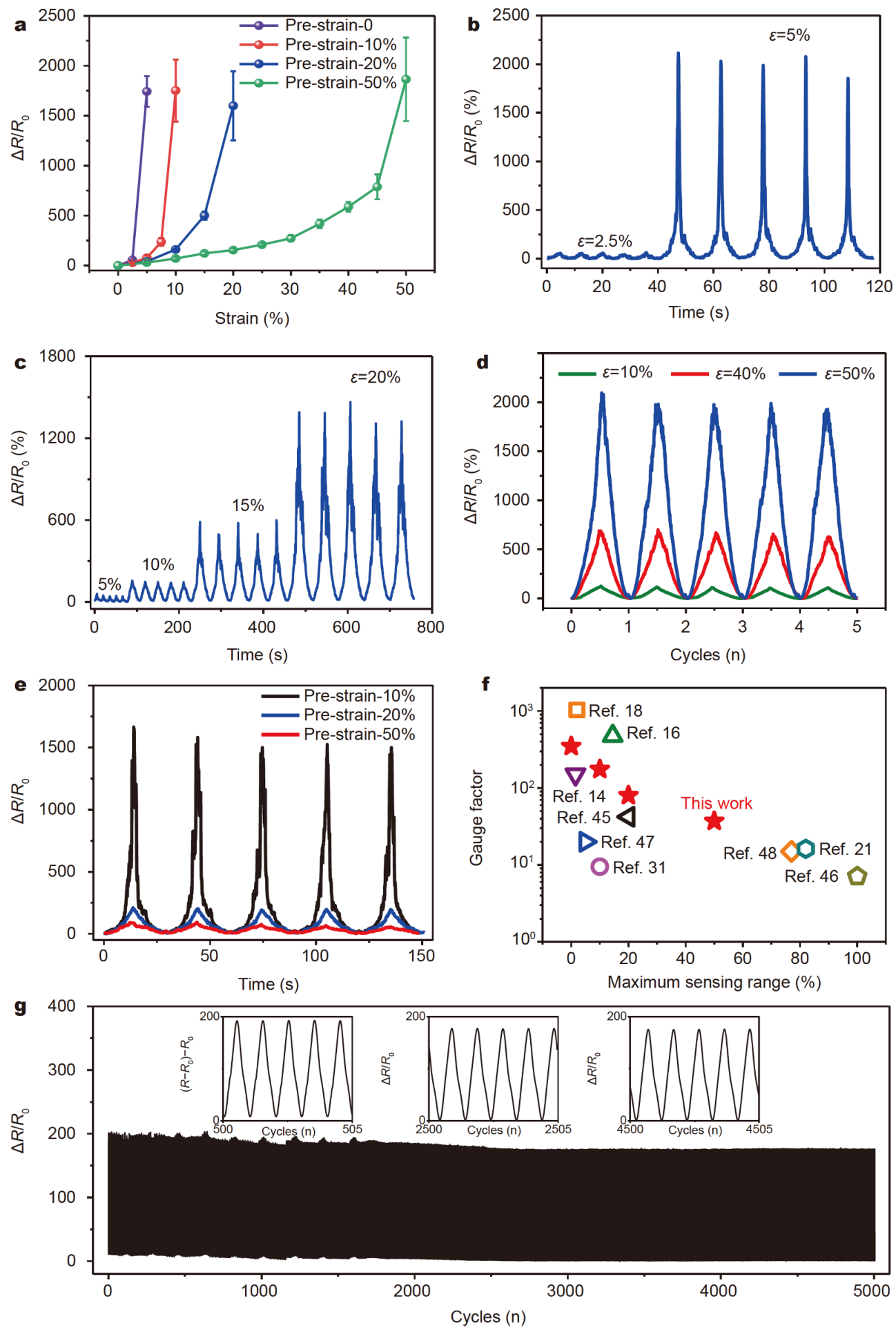

Figure 4 Performance of strain sensors based on the wrinkled graphene films. (a) Resistance changes of different strain sensors under increasing strain $(\varepsilon)$. Electrical responses of strain sensors based on pre-strain-0 (b) and pre-strain-20\% (c). (d) Cyclic resistance responses of strain sensors based on pre-strain-50\% under various strains. (e) Cyclic resistance responses of different strain sensors under 10\% tensile strain. (f) Comparison of GF and sensing range of the graphene-based strain sensors. (g) Stability test of the strain sensor (pre-strain-50\%) under $20 \%$ strain. Three insets are the enlarged figures of five cycles at different times. 

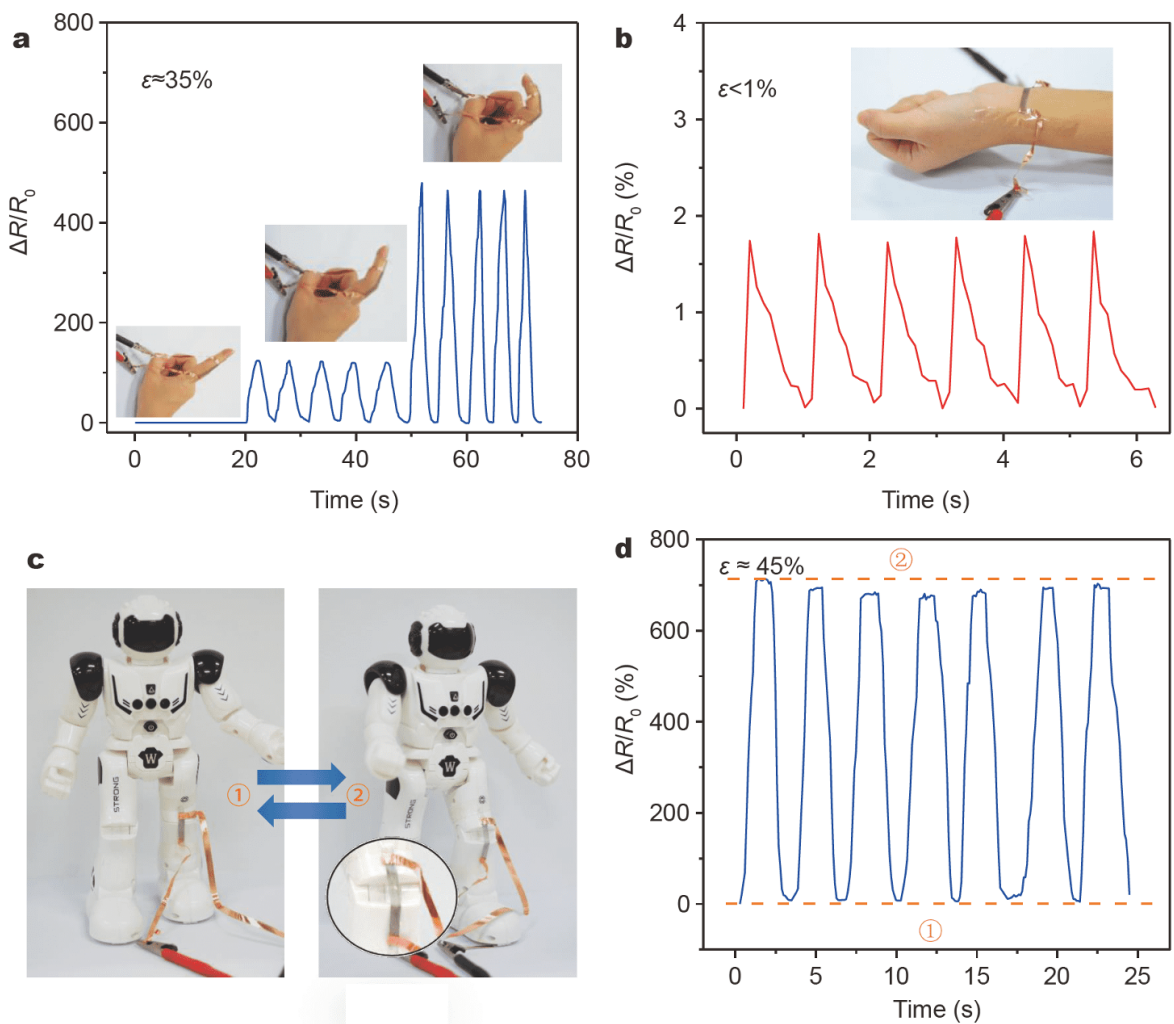

Figure 5 Practical applications of strain sensors. Real-time detection of finger motions (a) and surveillance of wrist pulses (b). (c) E-skins based on the strain sensor installed on the knee of a robot for detection of its erection and flexion status. (d) Corresponding cyclic electrical responses under two different statuses in (c).

\section{CONCLUSIONS}

In this work, we prepared the graphene film by solutionprocessed assembly of graphene sheets based on Marangoni effect, followed by the creation of uniaxial and periodic wrinkles in graphene film on a pre-stretched PDMS substrate. The width of the wrinkles can be controlled by changing the pre-strain of the PDMS substrate. Furthermore, the tension-induced changes of wrinkled morphology in double-layered graphene film have been clearly revealed. The mechanism study helps to fabricate the strain sensors based on wrinkled double-layered graphene with excellent working stability and tunable sensing performance, which is favorable to balance the sensitivity and the detection range. Our strain sensors are promising devices to be integrated into flexible electronics for motion monitoring, health surveillance, and artificial intelligence.

\section{Received 25 February 2020; accepted 22 March 2020;} published online 14 May 2020

1 Cai S, Xu X, Yang W, et al. Materials and designs for wearable photodetectors. Adv Mater, 2019, 31: 1808138
2 Xu K, Zhou R, Takei K, et al. Toward flexible surface-enhanced Raman scattering (SERS) sensors for point-of-care diagnostics. Adv Sci, 2019, 6: 1900925

3 Wang B, Facchetti A. Mechanically flexible conductors for stretchable and wearable E-skin and E-textile devices. Adv Mater, 2019, 31: 1901408

4 Chen W, Gui X, Yang L, et al. Wrinkling of two-dimensional materials: Methods, properties and applications. Nanoscale Horiz, 2019, 4: 291-320

5 Jian M, Wang C, Wang Q, et al. Advanced carbon materials for flexible and wearable sensors. Sci China Mater, 2017, 60: 10261062

6 Wang C, Xia K, Wang H, et al. Advanced carbon for flexible and wearable electronics. Adv Mater, 2019, 31: 1801072

7 Afroj S, Karim N, Wang Z, et al. Engineering graphene flakes for wearable textile sensors via highly scalable and ultrafast yarn dyeing technique. ACS Nano, 2019, 13: 3847-3857

8 Jung YH, Park B, Kim JU, et al. Bioinspired electronics for artificial sensory systems. Adv Mater, 2019, 31: 1803637

9 Huang H, Su S, Wu N, et al. Graphene-based sensors for human health monitoring. Front Chem, 2019, 7: 399

10 Zheng S, Zeng M, Cao H, et al. Insight into the rapid growth of graphene single crystals on liquid metal via chemical vapor deposition. Sci China Mater, 2019, 62: 1087-1095

11 Kim KS, Zhao Y, Jang H, et al. Large-scale pattern growth of graphene films for stretchable transparent electrodes. Nature, 2009, 
457: 706-710

12 Lee $\mathrm{Y}$, Bae S, Jang H, et al. Wafer-scale synthesis and transfer of graphene films. Nano Lett, 2010, 10: 490-493

13 Ma Y, Zheng Y, Zhu Y. Towards industrialization of graphene oxide. Sci China Mater, 2019, :

14 Hempel M, Nezich D, Kong J, et al. A novel class of strain gauges based on layered percolative films of $2 \mathrm{D}$ materials. Nano Lett, 2012, 12: 5714-5718

15 Jiang WS, Liu ZB, Xin W, et al. Reduced graphene oxide nanoshells for flexible and stretchable conductors. Nanotechnology, 2016, 27: 095301

16 Yang Z, Wang DY, Pang Y, et al. Simultaneously detecting subtle and intensive human motions based on a silver nanoparticles bridged graphene strain sensor. ACS Appl Mater Interfaces, 2018, 10: 3948-3954

17 Wang DY, Tao LQ, Liu Y, et al. High performance flexible strain sensor based on self-locked overlapping graphene sheets. Nanoscale, 2016, 8: 20090-20095

18 Li X, Yang T, Yang Y, et al. Large-area ultrathin graphene films by single-step Marangoni self-assembly for highly sensitive strain sensing application. Adv Funct Mater, 2016, 26: 1322-1329

19 Park Y, Shim J, Jeong S, et al. Microtopography-guided conductive patterns of liquid-driven graphene nanoplatelet networks for stretchable and skin-conformal sensor array. Adv Mater, 2017, 29: 1606453

20 Chae $\mathrm{SH}, \mathrm{Yu}$ WJ, Bae JJ, et al. Transferred wrinkled $\mathrm{Al}_{2} \mathrm{O}_{3}$ for highly stretchable and transparent graphene-carbon nanotube transistors. Nat Mater, 2013, 12: 403-409

21 Liu Q, Chen J, Li Y, et al. High-performance strain sensors with fish-scale-like graphene-sensing layers for full-range detection of human motions. ACS Nano, 2016, 10: 7901-7906

22 Chen W, Gui X, Liang B, et al. Controllable fabrication of largearea wrinkled graphene on a solution surface. ACS Appl Mater Interfaces, 2016, 8: 10977-10984

23 Zang J, Ryu S, Pugno N, et al. Multifunctionality and control of the crumpling and unfolding of large-area graphene. Nat Mater, 2013, 12: 321-325

24 Chen T, Xue Y, Roy AK, et al. Transparent and stretchable highperformance supercapacitors based on wrinkled graphene electrodes. ACS Nano, 2014, 8: 1039-1046

25 Thomas AV, Andow BC, Suresh S, et al. Controlled crumpling of graphene oxide films for tunable optical transmittance. Adv Mater, 2015, 27: 3256-3265

26 Lee WK, Kang J, Chen KS, et al. Multiscale, hierarchical patterning of graphene by conformal wrinkling. Nano Lett, 2016, 16: 71217127

27 Leem J, Wang MC, Kang P, et al. Mechanically self-assembled, three-dimensional graphene-gold hybrid nanostructures for advanced nanoplasmonic sensors. Nano Lett, 2015, 15: 7684-7690

28 Gabardo CM, Yang J, Smith NJ, et al. Programmable wrinkling of self-assembled nanoparticle films on shape memory polymers. ACS Nano, 2016, 10: 8829-8836

29 Chen PY, Sodhi J, Qiu Y, et al. Multiscale graphene topographies programmed by sequential mechanical deformation. Adv Mater, 2016, 28: 3564-3571

30 Yang YF, Tao LQ, Pang Y, et al. An ultrasensitive strain sensor with a wide strain range based on graphene armour scales. $\mathrm{Na}$ noscale, 2018, 10: 11524-11530

31 Tian H, Shu Y, Cui YL, et al. Scalable fabrication of high-performance and flexible graphene strain sensors. Nanoscale, 2014, 6:
699-705

32 Yun $\mathrm{T}$, Kim H, Iqbal A, et al. Electromagnetic shielding of monolayer MXene assemblies. Adv Mater, 2020, 32: 1906769

33 Kim HH, Lee SK, Lee SG, et al. Wetting-assisted crack- and wrinkle-free transfer of wafer-scale graphene onto arbitrary substrates over a wide range of surface energies. Adv Funct Mater, 2016, 26: 2070-2077

34 Feng C, Yi Z, Dumée LF, et al. Shrinkage induced stretchable micro-wrinkled reduced graphene oxide composite with recoverable conductivity. Carbon, 2015, 93: 878-886

$35 \mathrm{Chu} \mathrm{JH}$, Lee $\mathrm{DH}$, Jo J, et al. Highly conductive and environmentally stable organic transparent electrodes laminated with graphene. Adv Funct Mater, 2016, 26: 7234-7243

36 Takei A, Jin L, Fujita $\mathrm{H}$, et al. High-aspect-ratio ridge structures induced by plastic deformation as a novel microfabrication technique. ACS Appl Mater Interfaces, 2016, 8: 24230-24237

37 Ahadian S, Estili M, Surya VJ, et al. Facile and green production of aqueous graphene dispersions for biomedical applications. $\mathrm{Na}$ noscale, 2015, 7: 6436-6443

38 Bae SH, Lee Y, Sharma BK, et al. Graphene-based transparent strain sensor. Carbon, 2013, 51: 236-242

39 Zhu W, Low T, Perebeinos V, et al. Structure and electronic transport in graphene wrinkles. Nano Lett, 2012, 12: 3431-3436

40 Feng C, Yi Z, Dumée LF, et al. Tuning micro-wrinkled graphene films for stretchable conductors of controllable electrical conductivity. Carbon, 2018, 139: 672-679

41 Rolnick H. Tension coefficient of resistance of metals. Phys Rev, 1930, 36: 506-512

42 Pang Y, Tian H, Tao L, et al. Flexible, highly sensitive, and wearable pressure and strain sensors with graphene porous network structure. ACS Appl Mater Interfaces, 2016, 8: 26458-26462

43 Park JJ, Hyun WJ, Mun SC, et al. Highly stretchable and wearable graphene strain sensors with controllable sensitivity for human motion monitoring. ACS Appl Mater Interfaces, 2015, 7: 63176324

44 Wang Y, Wang Y, Yang Y. Graphene-polymer nanocompositebased redox-induced electricity for flexible self-powered strain sensors. Adv Energy Mater, 2018, 8: 1800961

45 Chun S, Choi Y, Park W. All-graphene strain sensor on soft substrate. Carbon, 2017, 116: 753-759

46 Yan C, Wang J, Kang W, et al. Highly stretchable piezoresistive graphene-nanocellulose nanopaper for strain sensors. Adv Mater, 2014, 26: 2022-2027

47 Liu Q, Zhang M, Huang L, et al. High-quality graphene ribbons prepared from graphene oxide hydrogels and their application for strain sensors. ACS Nano, 2015, 9: 12320-12326

48 Jeong YR, Park H, Jin SW, et al. Highly stretchable and sensitive strain sensors using fragmentized graphene foam. Adv Funct Mater, 2015, 25: 4228-4236

Acknowledgements This work was financially supported by the National Natural Science Foundation of China (51772335), and the Science and Technology Program of Guangzhou (201904010450).

Author contributions Jia Y and Chen W directed the research. Ye C performed the graphene synthesis under the supervision of Lin CT. Gui $\mathrm{X}$ supervised the project and conceived the experiments. Yang R, Yang $\mathrm{L}$, and $\mathrm{Hu} \mathrm{Q}$ carried out samples transfer and performed the tests such as SEM, Raman. Zhang $Z$ and Liang $B$ carried out the mechanicalelectrical measurements. Jia Y, Chen W, Yang BR, Tang Z, Lin CT and 
Gui X analyzed the experimental data, designed the figures and co-wrote the manuscript. All authors contributed to the general discussion.

Conflict of interest The authors declare no conflict of interest.

Supplementary information Supporting data are available in the online version of the paper.

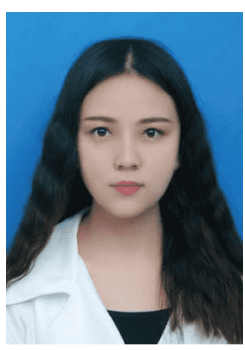

Yufei Jia received her Bachelor's degree from Hefei University of Technology in 2017. She is a postgraduate student at Sun Yat-sen university now. Her research interest is graphene-based strain sensors.

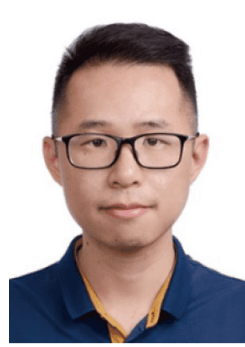

Wenjun Chen obtained his $\mathrm{PhD}$ majored in condensed matter physics at Sun Yat-sen University in 2018. Now he is working as a Postdoctoral researcher at Tsinghua-Berkeley Shenzhen Institute (TBSI), Tsinghua University. His research is focused on the preparation of $2 \mathrm{D}$ materials for applications in flexible sensors.

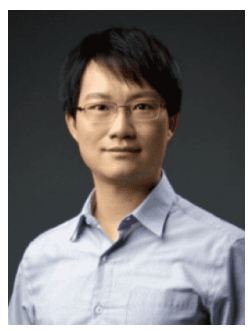

Cheng-Te Lin received his $\mathrm{PhD}$ degree in materials science and engineering at Tsing Hua University (Hsin-Chu) in 2008. In 2012, he was a postdoc at Massachusetts Institute of Technology (MIT, USA). Since 2014 June, he is working as a full professor at Ningbo Institute of Material Technology and Engineering. His research interests focus on the development of graphenebased applications, including functional composites, thermal interface materials, and biosensors.

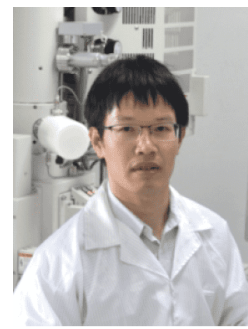

Xuchun Gui received his $\mathrm{PhD}$ degree in materials science and engineering at Tsinghua University in 2011. Then, He joined Sun Yat-Sen University as an assistant professor in 2011, and appointed as an associate professor in 2014. From 2014 to 2015 he worked as a visiting professor at The Hong Kong University of Science and Technology. His research interests focus on the synthesis of carbon nanomaterials and 2D materials, and their applications in flexible optoelectronics and sensor devices.

\section{基于马兰戈尼效应制备具有周期性褶皱结构的石 墨烯薄膜及其在高灵敏应变探测中的应用}

贾宇飞 ${ }^{1 \dagger}$, 陈文骏 ${ }^{1 \dagger}$, 叶辰 ${ }^{2}$, 杨㭉亮 ${ }^{1}$, 杨蕾蕾 ${ }^{1}$, 张子安 ${ }^{1}$, 胡清梅 $^{1}$, 梁秉豪 ${ }^{1}$, 杨柏儒, 汤子康 ${ }^{1,3}$, 林正得 ${ }^{2}$, 桂许春 $^{1^{*}}$

摘要 石墨烯薄膜中可控的微纳结构, 有利于调控其物理性质并拓 宽其在柔性电子器件中的应用. 近年来, 研究人员致力于控制石墨 烯薄膜中褶皱、起伏波纹等微纳结构的形成. 但是, 在石墨烯薄膜 中可控地形成有序的褶皱状结构仍然面临巨大挑战. 本文报道了 一种简单地制备具有周期性平行褶皱结构的石墨烯薄膜的方法, 即通过将溶液表面自组装形成的石墨烯薄膜转移至预拉伸的聚二 甲基硅氧烷(PDMS)基底上而得到. 制备的石墨烯薄膜中, 褶皱的宽 度可以简单地通过改变基底的预拉伸形变来控制. 当PDMS基底预 拉伸应变分别为 $10 \%$ 、20\%和 $50 \%$ 时，薄膜中褶皱的平均宽度分别 为3.68、2.99和 $2.01 \mu \mathrm{m}$. 本文还进一步研究和分析了双层堆叠、褶 皱状石墨烯薄膜, 在拉伸形变时的形貌结构变化. 此外, 本文基于该 褶皱状石墨烯薄膜, 构建了应变传感器. 该传感器展现出高灵敏 度、宽探测范围和优良的循环稳定性，其在实时运动探测、健康 监测和电子皮肤等领域有着广阔应用前景. 